\title{
UNA PROPUESTA DE INTERVENCIÓN PARA NIÑOS CON TRASTORNO DEL ESPECTRO AUTISTA A TRAVÉS \\ DE LA LECTURA DIALÓGICA Y LA REALIDAD AUMENTADA
}

\author{
A PROPOSAL OF INTERVENTION FOR CHILDREN WITH AUTISM \\ SPECTRUM DISORDER THROUGH DIALOGICAL READING \\ AND AUGMENTED REALITY
}

\section{Inmaculada Baixauli-Fortea ${ }^{a}$, Soledad Gómez-García ${ }^{b *}$, María de El Puig Andrés-Sebastiáb y Carmen Berenguer-Forner ${ }^{c}$}

Fechas de recepción y aceptación: 31 de julio de 2019, 6 de diciembre de 2019

Resumen: Los niños con trastorno del espectro autista (TEA) requieren intervenciones educativas basadas en la evidencia que traten las dificultades en la atención conjunta, la reciprocidad social, la comunicación, las habilidades mentalistas o el funcionamiento ejecutivo, al tiempo que consideren sus fortalezas en el procesamiento visual y su estilo de aprendizaje. Una intervención que reúne estas cualidades es la técnica de la lectura dialógica de cuentos, como sugieren recientes investigaciones con resultados prometedores. Además, su combinación con nuevas tecnologías como la realidad aumentada puede incrementar la motivación de los niños hacia la tarea y favorecer el aprendizaje. En este artículo se presenta una propuesta de intervención consistente en una serie de cuentos enriquecidos con RA. Los cuentos han sido diseñados con objeto de abordar déficits nucleares en el TEA. Se incluyen también algunas orientaciones para su aplicación, de acuerdo con la técnica de la lectura dialógica.

Palabras clave: trastorno del espectro autista, lectura dialógica, realidad aumentada, intervención.

a Facultad de Psicología. Universidad Católica de Valencia San Vicente Mártir.

${ }^{\text {b }}$ Departamento de Didáctica General, Teoría de la Educación e Innovación Educativa. Facultad de Magisterio y Ciencias de la Educación. Universidad Católica de Valencia San Vicente Mártir.

${ }^{*}$ Correspondencia: Universidad Católica de Valencia San Vicente Mártir. Facultad de Magisterio y Ciencias de la Educación. Calle Sagrado Corazón, 5. 46110 (Godella), Valencia. España.

E-mail: soledad.gomez@ucv.es

c Departamento de Psicología Evolutiva y de la Educación. Universidad de Valencia. 
Abstract: Children with Autism Spectrum Disorders (ASD) require evidenced-based educational interventions that address their impairments in joint attention, social reciprocity, communication, theory of mind or executive functioning, while considering their strengths on visual processing and their learning style. An approach that meets these qualities is the dialogic reading technique, as recent research shows. Furthermore, its combination with new technologies like augmented reality may increase children's motivation towards the task and promote the learning process. In this article, a proposal of intervention is presented which consists of a series of stories, enhanced by augmented reality. The stories have been designed in order to target core disabilities in ASD. Some guidelines for its implementation are also provided, according to the dialogic reading technique.

Keywords: autism spectrum disorder, dialogic reading, augmented reality, intervention.

\section{INTRODUCCIÓN}

El trastorno del espectro autista (TEA) es una condición del neurodesarrollo caracterizada por dificultades persistentes en la comunicación y la interacción social, unido a una restricción en los intereses y a la presencia de conductas repetitivas (APA, 2013). En los últimos años, su prevalencia se ha incrementado notablemente, llegando a configurarse como el trastorno neuroevolutivo con un ritmo de crecimiento más rápido. En 2018, aproximadamente, 1 de cada 59 niños fueron diagnosticados con TEA en Estados Unidos, de acuerdo con las estimaciones del Center for Disease Control and Prevention (Autism and Developmental Disabilities Monitoring Network, USA).

Las personas con TEA, con su peculiar estilo de procesar la información y su original manera de percibir e interpretar el mundo, suponen un reto a todas las personas encargadas de su educación, quienes deben adaptar el estilo de enseñanza y las estrategias pedagógicas a su perfil de funcionamiento cognitivo (González et al., 2014). La población creciente de niños con TEA requiere, por tanto, intervenciones educativas basadas en la evidencia que traten sus variadas necesidades al tiempo que maximicen su potencial.

En el ámbito educativo, las denominadas habilidades de lectura emergente (emergent literacy) se refieren a las experiencias de lectura y escritura de niños pequeños antes de su aprendizaje convencional (Teale y Sulzby, 1986). En el marco de estas prácticas se encuentra la lectura compartida que, en general, describe el acto en el que los adultos leen en voz alta a los niños, mientras estimulan la interacción a través de la formulación de preguntas o la impli- 
cación en explicaciones y comentarios sobre el libro (Hudson y Test, 2011). Concretamente, la lectura dialógica es un método particular de lectura compartida en la que el adulto usa una serie de ayudas específicas para estimular la participación de los niños durante la lectura de libros. Mientras el adulto lee al niño, le incita a involucrarse en la actividad, evalúa la corrección de sus contribuciones y expande sus enunciados. Los tipos específicos de ayudas usadas en la lectura dialógica incluyen la finalización de frases, el recuerdo de información relevante, la realización de preguntas abiertas y el planteamiento de cuestiones vinculadas con la experiencia del niño. Con el tiempo, el apoyo del adulto disminuye a medida que el niño desempeña un rol cada vez más activo (Whitehurst et al., 1994). Esta aproximación ha demostrado ser efectiva en la mejora del lenguaje y las habilidades de lectura emergente debido a que los niños están activamente implicados en la tarea y reciben refuerzo por su participación, mientras que el feedback proporcionado por el adulto sirve como modelo adecuado de lenguaje. Una de las claves de su éxito reside, además, en la creación de una interacción responsiva entre el adulto y el niño, lo que ha demostrado tener un efecto positivo en el desarrollo del lenguaje y en la lectura temprana (Justice, Mashburn, Hamre y Pianta, 2008). De hecho, la eficacia de la lectura dialógica se ha documentado extensamente en el desarrollo del lenguaje expresivo y ha sido considerada una práctica recomendada en la enseñanza de la lectura emergente por What Works Clearinghouse (US Department of Education, 2007). Asimismo, el National Early Literacy Panel (2008) refirió ganancias con tamaño moderado del efecto en el lenguaje oral y conocimiento de lo impreso en niños tanto con desarrollo típico como con trastornos del lenguaje que habían seguido este enfoque. También ha logrado mejorar el rendimiento de niños preescolares con trastornos del lenguaje en medidas de lenguaje literal e inferencial (van Kleeck, Vander Woude y Hammett, 2006) y vocabulario (Penimonti y Justice, 2010), lo que puede contribuir al rendimiento lector posterior (Cunningham y Zibulsky, 2011).

\subsection{La intervención mediante lectura dialógica en niños con TEA}

Los niños con TEA pueden tener dificultades para participar en la lectura compartida de libros debido, en parte, a las demandas sociales y comunicativas 
de la tarea. Investigaciones previas indican que atienden durante menos tiempo a este tipo de actividades (Watson, Andrews y Orovitz, 1996), suelen demandarlas con menor frecuencia y las disfrutan menos, en comparación con niños con desarrollo típico equiparados en nivel de lenguaje (Lanter, Watson, Erickson y Freeman, 2012). La lectura compartida implica el desarrollo de una serie de habilidades en las que los niños con TEA tienen dificultades, como son la atención conjunta, la reciprocidad social, el lenguaje y la comunicación, las habilidades mentalistas y el funcionamiento ejecutivo. Estas habilidades son, precisamente, objeto de intervención a través de la lectura dialógica.

Las rutinas de lectura dialógica proporcionan oportunidades para la atención conjunta (Penimonti y Justice, 2010), un objetivo instruccional importante para los niños pequeños con TEA, que desde la infancia temprana tienen dificultades para coordinar la atención entre las personas y los objetos (Dawson et al., 2004). Específicamente, la atención conjunta incluye habilidades tales como orientarse y atender al interlocutor, alternar la mirada entre las personas y los objetos y compartir el afecto con otra persona (Wetherby y Prizant, 2002). En este sentido, la lectura compartida de libros requiere que los niños sean capaces de mantener la interacción social en torno a un tema concreto y hacer preguntas o comentarios sobre lo que se observa.

Asimismo, los niños con TEA inician con menor frecuencia comentarios o preguntas (Freeden y Koegel, 2006) y en su comunicación predominan los actos con función regulativa o instrumental sobre aquellos dirigidos a compartir socialmente una actividad (Wetherby, Prizant y Schuler, 2000). En este sentido, la lectura dialógica es una tarea social (Penimonti y Justice, 2010) que tiene como finalidad estimular la participación activa del niño de tal forma que desarrolle junto con el adulto una conversación sobre el libro, lo que implica iniciaciones sociales y respuestas por parte de ambos.

Estrechamente relacionadas con las capacidades de referencia conjunta, se encuentran las habilidades de lenguaje expresivo y receptivo, área también deficitaria en el TEA (Tager-Flusberg, Paul y Lord, 2005). Las intervenciones en lectura dialógica podrían paliar estos déficits a través de una instrucción facilitadora de la adquisición del lenguaje (Goldstein, 2002; Justice, Mashburn, Hamre y Pianta, 2008). En particular, los niños con TEA experimentan dificultades significativas en la pragmática, particularmente, en la competen- 
cia conversacional y narrativa (Baixauli, Colomer, Roselló y Miranda, 2016). Los déficits narrativos parecen estar directamente vinculados con síntomas nucleares del trastorno (Stirling, Douglas, Leekam y Carey, 2014), como son las dificultades en la integración de la información desde diversas fuentes o la excesiva atención a los detalles, a expensas de los aspectos relevantes, tal y como sostiene la teoría de la coherencia central débil (Happé y Frith, 2006). En este sentido, la lectura dialógica expone al niño a un input lingüístico ajustado, mientras el adulto le proporciona el andamiaje suficiente para el desarrollo apropiado de la narración.

Otra área de vulnerabilidad en el TEA la constituyen los déficits en la teoría de la mente, es decir, la capacidad para inferir estados mentales (Baron-Cohen, Leslie y Frith, 1985) y para empatizar (Baron-Cohen, 2010), lo que puede afectar a la interacción social y a la comprensión inferencial de las historias (Tager-Flusberg, Paul y Lord, 2005). Precisamente, la lectura dialógica requiere respuestas basadas en el reconocimiento de emociones y estados cognitivos, en la comprensión de lo literal y lo inferencial. Asimismo, las dificultades en las funciones ejecutivas (atención, organización, planificación, flexibilidad cognitiva, autorregulación) han sido consideradas una de las causas fundamentales de las alteraciones cognitivas en el TEA (Ozonoff, Pennington y Rogers, 1991). La lectura dialógica incide en aspectos claramente relacionados con el funcionamiento ejecutivo como la secuenciación de sucesos o la atención sostenida y selectiva.

Por último, en cualquier intervención educativa, el método de instrucción y las características de los materiales empleados deben considerar las potencialidades de sus destinatarios. En este sentido, la lectura dialógica se adecua a las fortalezas de las personas con TEA en el procesamiento de la información, ya que se caracteriza por un alto grado de estructuración y se pueden utilizar las ilustraciones de los libros como apoyos visuales. Los niños con TEA se benefician de rutinas que ocurren regularmente, incluyen roles claramente definidos, incorporan scripts y tienen una estructura facilitadora (Earles-Vollrath, Cook, Robbins y Ben-Arieh, 2008). Estas características son inherentes a la técnica de la lectura dialógica. Además, las personas con TEA son aprendices visuales, es decir, comprenden mejor la información presentada por la vía visual (Thaut, 1987). Las imágenes son estables y permanecen en el tiempo; en contraposición, la palabra hablada desaparece rápidamente. 
Por este motivo, las ilustraciones y otros soportes visuales constituyen elementos esenciales en este tipo de intervención.

A pesar de todos los indicadores que sugieren que la lectura dialógica puede ser una técnica muy útil en la intervención en niños con TEA, el número de investigaciones sobre el tema es muy reducido, si bien los escasos estudios preliminares aportan resultados prometedores. Así, se ha demostrado que los niños con TEA son capaces de participar con éxito en la lectura compartida de historias cuando se realizan adaptaciones a la actividad. Las acomodaciones incluyen el uso de claves visuales (Mucchetti, 2013), la música (Carnahan, Basham y Musti-Ra, 2009) y las estrategias de andamiaje (procedimientos de cloze, elecciones binarias, preguntas Qu-, y expansiones Bellon-Harn, Ogletree y Harn, 2000). Los resultados revelan mejoras en la conducta centrada en la tarea (on-task behavior) (Carnahan et al., 2009), disminución de las ecolalias (Bellon-Harn et al., 2000), progresos en la comprensión de la historia (Mucchetti, 2013) y mayor participación en la actividad (Fleury, Herriott-Miramontez, Hudson, y Schwartz, 2014). Estos alentadores hallazgos llevaron a Whalon, Martínez, Shannon, Butcher y Hanline (2015) a realizar una adaptación de la lectura dialógica a los estudiantes con TEA, mediante un procedimiento que denominaron RECALL (Reading to Engage Children with Autism in Language and Literacy). Como resultado de la intervención, los niños mejoraron su habilidad para responder a una variedad de preguntas sobre la historia tanto de tipo factual como inferencial.

\subsection{La incorporación de la tecnología en la intervención en el TEA: la realidad aumentada}

La investigación ha sugerido combinar la lectura dialógica con la tecnología para mejorar los resultados obtenidos en la atención y el lenguaje de preescolares con TEA (Alison, Root, Browder y Wood, 2017). De acuerdo con esta línea de trabajo, avances tecnológicos como la realidad aumentada (RA) pueden configurarse como una herramienta excelente para facilitar el aprendizaje en esta población. La RA no reemplaza el mundo real por uno virtual, sino que lo complementa. El usuario nunca pierde el contacto con el mundo real que tiene al alcance de su vista y al mismo tiempo puede interactuar con 
la información virtual superpuesta (Basogain, Olabe, Espinosa, Rouèche y Olabe, 2007). Por este motivo, la RA permite el diseño de materiales educativos atractivos que, al mismo tiempo, pueden ser utilizados en situaciones que resulten beneficiosas para alumnos con necesidades especiales (Lin y Chao, 2010).

En el caso concreto de los niños con TEA, en consonancia con su estilo de aprendizaje y el interés por los estímulos que se presentan visualmente, la RA puede convertirse en un medio para reconducir los déficits anteriormente descritos. Por ejemplo, mediante la intensificación de determinadas claves visuales en las historias, la RA puede ayudar a los niños con autismo a focalizar y mantener su atención así como favorecer su motivación hacia el aprendizaje (Karamanoli, Tsinakos y Karagiannidis, 2017). La RA, como complemento al uso de imágenes estáticas o fragmentadas, puede contrarrestar los déficits en la coherencia central y en la atención facilitando el procesamiento de los estímulos más relevantes de las escenas de una historia; entre ellos, el reconocimiento y comprensión de emociones, causas y consecuencias que, como se ha referido anteriormente, constituye un área claramente afectada en esta población. De este modo, esta tecnología ofrece una serie de ventajas para el proceso de aprendizaje: permite resaltar visualmente determinados aspectos de la realidad, como los estímulos sociales importantes, y propicia una representación corporal, permitiendo visualmente seguir los movimientos y ser conscientes de las acciones de los elementos animados (Escribano, Herrera, Jordan y Gimeno, 2010).

Hasta ahora la RA ha sido empleada de forma incipiente con resultados muy positivos en la intervención en niños TEA en el área del juego (Bai, Blackwell y Coulouris, 2015), y en la atención sostenida y selectiva durante tareas desarrolladas en sesiones terapéuticas (Escobedo, Tentori, Favela y García-Rosas, 2014). También se ha constatado la mejora del procesamiento de claves faciales no verbales en el marco de historias, utilizando una combinación de RA y videomodelado (Chen, Lee y Lin, 2016).

La propuesta de intervención que presentamos a continuación se basa en la RA unida a la técnica de la lectura dialógica, que puede ser un elemento importante en la mejora de los recursos atencionales y el interés y motivación del niño hacia la tarea. 


\section{DesCRIPCIÓN DE LA PROPUESTA}

\subsection{Los materiales y contenidos}

Los materiales base de la intervención lo conforman tres cuentos protagonizados por un mismo personaje, que relatan acontecimientos cotidianos en la vida de un niño (un cumpleaños, una excursión al campo y un día en la playa). Los cuentos siguen el esquema de la gramática de la historia en lo que a componentes se refiere (Stein y Glenn, 1979). Se basan en un número reducido de episodios (entre cuatro y cinco), compuestos por localización, suceso desencadenante, plan, consecuencia, respuesta interna y resolución. El lenguaje de cada cuento es sencillo desde el punto de vista sintáctico y léxico, y las ilustraciones son claras e incluyen pocos elementos. Con ello se ha pretendido evitar la sobreestimulación, que puede dificultar el procesamiento de la información.

En cada cuento se trabajan habilidades de teoría de la mente (ToM) como emociones básicas (contento, asustado, tristeza, dolor, llorar) y complejas (confuso, aliviado, desconcertado, consolar, satisfacción), falsa creencia, cogniciones (decidir, imaginar) y planes dirigidos a metas. Se ha tenido especial cuidado en seguir una gradación en la complejidad de las habilidades de ToM, de acuerdo con la perspectiva evolutiva descrita en Wellman y Lagattuta (2000). Las escenas de contenido mentalista han sido elaboradas en formato de RA, a fin de enfatizar la habilidad correspondiente y aumentar la motivación del niño hacia su aprendizaje. Para el acceso a la RA se usa como soporte tecnológico una tableta, ya que los cuentos disponen de una aplicación ( $a p p$ ) asociada. Además, cada historia incluye también una serie de preguntas de comprensión literal e inferencial. Las figuras 1 y 2 representan distintas escenas que suscitan la descripción de habilidades mentalistas. En la figura 3 puede observarse un ejemplo de una escena 2D para enfatizar la emoción de contento; de forma paralela, en la figura 4 se puede observar cómo se mostraría dicha escena en $3 \mathrm{D}$, al ser visualizada usando la app de $\mathrm{RA}$ mediante una tableta. 
FIGURA 1

El cumpleaños de Ismael

Habilidades de ToM: creer, imaginar

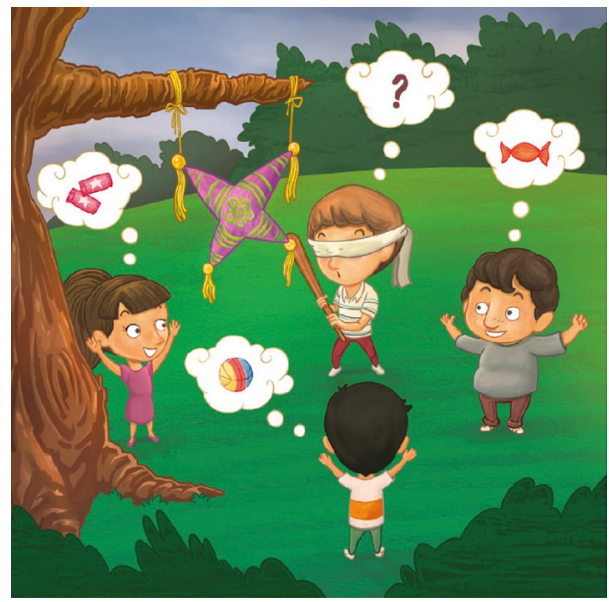

FIGURA 3

El cumpleaños de Ismael

Ejemplo del libro en 2D.

Emoción: contento

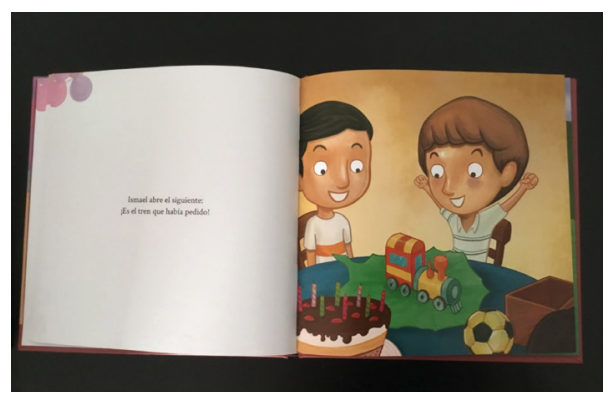

FIGURA 2

Ismael va a la playa

Habilidades de ToM: creer, sorprendersel

asustarse

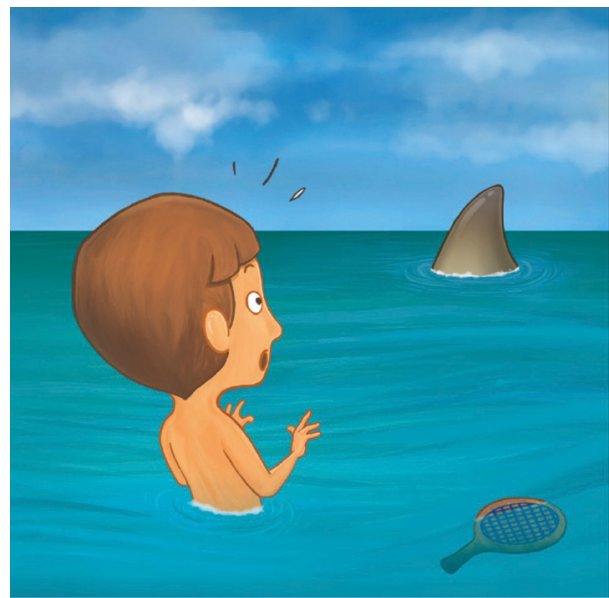

FIGURA 4

El cumpleaños de Ismael

Ejemplo de la ilustración en 3D con RA.

Emoción: contento

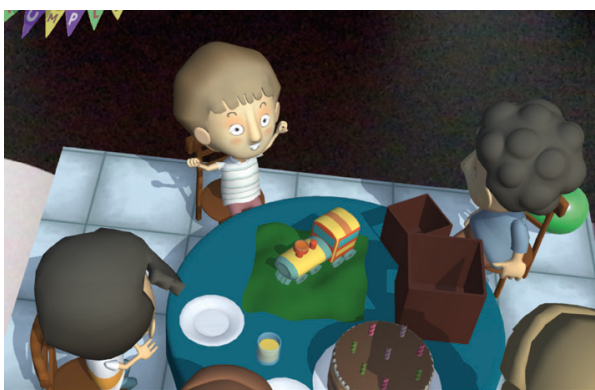

A través de un vídeo, disponible en la siguiente URL, https://youtu.be/ aVDtQ1ms zY, se puede acceder a un ejemplo de los materiales. El vídeo muestra la ejecución de la aplicación de tableta cuando se activan las escenas usando este tipo de dispositivos. 


\subsection{La metodología de intervención: la técnica de la lectura dialógica}

La intervención en cada cuento ha sido manualizada siguiendo las orientaciones de la lectura dialógica adaptada a estudiantes con autismo (RECALL, Whalon, Delano y Hanline, 2013; Whalon, Martínez, Shannon, Butcher y Hanline, 2015). RECALL integra las ayudas de la lectura dialógica, identificadas con las siglas CROWD (Completion, Recall, Open-Ended, WhQuestions y Distancing) (véase tabla 1) junto a guías para elicitar la atención conjunta y las iniciaciones. Además, se añaden cuatro tipos de ayuda más: identificación de la emoción, captación de la atención, pausa intencional e iniciación. La identificación de la emoción requiere que el niño identifique cómo se está sintiendo un personaje. La ayuda de captación de la atención se usa para establecer la atención conjunta. Para estimular la iniciación, se realiza una pausa intencional. Si es necesario, se ayuda explícitamente a los niños a iniciar una interacción.

TABLA 1

Ayudas CROWD (Completion, Recall, Open-ended, Wh-questions, Distancing)

\begin{tabular}{|l|l|l|}
\hline \multicolumn{1}{|c|}{ Ayuda } & \multicolumn{1}{|c|}{ Definición } & \multicolumn{1}{c|}{ Ejemplo } \\
\hline $\begin{array}{l}\text { Inducción terminati- } \\
\text { va (Completion) }\end{array}$ & $\begin{array}{l}\text { Se realiza una pausa al final de una oración y } \\
\text { el niño la completa. }\end{array}$ & “Y entonces Ismael...” \\
\hline $\begin{array}{l}\text { Recuerdo de infor- } \\
\text { mación (Recall) }\end{array}$ & $\begin{array}{l}\text { Preguntas sobre lo que ocurrió en el libro. } \\
\text { Las preguntas se realizan sobre la trama o la } \\
\text { secuencia de sucesos. }\end{array}$ & “QQué hizo Ismael?” \\
\hline $\begin{array}{l}\text { Pregunta de respuesta } \\
\text { abierta (Open-ended) })\end{array}$ & $\begin{array}{l}\text { Se solicita al niño que diga lo que está ocu- } \\
\text { rriendo en una imagen. }\end{array}$ & “QQué está pasando aquí?” \\
\hline $\begin{array}{l}\text { Preguntas Qu- } \\
\text { (Wh-questions) }\end{array}$ & $\begin{array}{l}\text { Las preguntas Qu- empiezan con qué, dónde, } \\
\text { cuándo, por qué y cómo. }\end{array}$ & $\begin{array}{l}\text { “Qué es esto?” (señalando } \\
\text { un objeto en el libro). }\end{array}$ \\
\hline $\begin{array}{l}\text { Distanciamiento } \\
\text { (Distancing) }\end{array}$ & $\begin{array}{l}\text { Preguntas que ayudan al niño a relacionar la } \\
\text { información en el libro con sus propias expe- } \\
\text { riencias. }\end{array}$ & $\begin{array}{l}\text { “Te ha pasado algo así algu- } \\
\text { na vez?” }\end{array}$ \\
\hline
\end{tabular}

Nota: Adaptado de Zevengergen y Whitehurst (2003, p. 180). 


\section{VİAS FUTURAS DE INVESTIGACIÓN}

En este artículo ${ }^{1}$ se ha presentado el diseño y fundamentación de una propuesta de intervención dirigida a niños con TEA a través de la lectura dialógica. La propuesta permite abordar distintas áreas deficitarias en esta población, como son la atención conjunta, la reciprocidad social, la comunicación, la teoría de la mente o el funcionamiento ejecutivo. Este es un proyecto innovador en el que se espera que el enriquecimiento con tecnología RA ayude a captar la atención del niño y aumentar la motivación hacia el aprendizaje. Un objetivo inmediato es la realización de estudios exploratorios que permitan calibrar la viabilidad y eficacia de la intervención. Estas investigaciones deben considerar su impacto en medidas lingüísticas (vocabulario, narración), el desarrollo de habilidades mentalistas, la implicación en la tarea (engagement) o la participación verbal del niño en términos de iniciaciones espontáneas y respuestas a las preguntas del adulto. Otra cuestión importante es la aplicabilidad de la propuesta al formato grupal, en el contexto del aula, desde la perspectiva de la inclusión educativa, dado que este recurso puede beneficiar no solo a los niños con TEA, sino a todo el alumnado. Esperamos que su implementación pueda contribuir a mejorar las dificultades que afrontan las personas con TEA y ayude a promover su pleno desarrollo.

\section{Bibliografía}

Alison, C., Root, J. R., Browder, D. M. y Wood, L. (2017). TechnologyBased Shared Story Reading for Students With Autism Who Are EnglishLanguage Learners. Journal of Special Education Technology 32(2), 91101.

American Psychiatric Association (APa) (2013). Diagnostic and statistical manual of mental disorders (5. a edición). Washington DC: American Psychiatric Association.

\footnotetext{
${ }^{1}$ Este artículo es fruto del proyecto de Investigación realizado con fondos obtenidos del proyecto UCV2017-2018-46, Convocatoria de Ayudas de la Universidad Católica San Vicente Mártir para el desarrollo de Proyectos de Investigación 2017-2018.
} 
Bai, Z., Blackwell, A. y Coulouris, G. (2015). Using augmented reality to elicit pretend play for children with autism. IEE Transactions on visualization and computer graphics 21 (5), 598-610.

Baixauli, I., Colomer, C., Roselló, B. y Miranda, A. (2016). Narratives of children with high-functioning autism spectrum disorder: A meta-analysis. Research in Developmental Disabilities 59, 234-254.

BAron-Cohen, S. (2010). Empathizing, systemizing, and the extreme male brain theory of autism. Progress in Brain Research 186, 167-175.

Baron-Cohen, S., Leslie, A. M. y Frith, U. (1985). Does the autistic child have a "theory of mind"? Cognition 21(1), 37-46.

Basogain, X., Olabe, M., Espinosa, C., Rouèche, C. y Olabe, J. C. (2007). Realidad aumentada en la educación: una tecnología emergente. Actas de Online Educa Madrid 2007: 7. ${ }^{a}$ Conferencia Internacional de la Educación y la Formación basada en las Tecnologías (pp. 24-29).

Bellon, M. L., Ogletree, B. T. y Harn, W. E. (2000). Repeated Storybook Reading as a Language Intervention for Children with Autism: A Case Study on the Application of Scaffolding. Focus on Autism and Other Developmental Disabilities 15(1), 52-58.

Carnahan, C., Basham, J. y Musti-Ra, S. (2009). A low-technology strategy for increasing engagement of students with autism and significant learning needs. Exceptionality 17(2), 76-87.

Chen, C. H., Lee, I. J. y Lin, L. Y. (2016). Augmented reality-based videomodeling storybook of nonverbal facial cues for children with autism spectrum disorder to improve their perceptions and judgments of facial expressions and emotions. Computers in Human Behavior 55, 477-485.

Cunningham, A. E. y Zibulsky, J. (2011). Tell Me a Story: Examining the Benefits of Shared Reading, en S. B. Neuman y D. K. Dickinson (eds.), Handbook of Early Literacy Research (vol. 3, pp. 396-411). Nueva York: The Guilford Press.

Dawson, G., Toth, K., Авbott, R., Osterling, J., Munson, J., Estes, A. y LiAw, J. (2004). Early social attention impairments in autism: social orienting, joint attention, and attention to distress. Developmental Psychology 40(2), 271-283.

Earles-Vollrath, T. L., Cook, K. T., Robins, L. y Ben-Arieh, J. (2008). Instructional strategies to facilitate successful learning outcomes for students 
with autism spectrum disorders, en R. L. Simpson y B. S. Myles (eds.), Educating children and youth with autism: Strategies for effective practice (2. ${ }^{\text {a }}$ edición, pp. 222-243). Austin, TX: Pro-ED.

Escobedo, L., Tentori, M., Quintana, E., Favela, J. y García-Rosas, D. (2014). Using augmented reality to help children with autism stay focused. IEEE Pervasive Computing 13(1), 38-46.

Escribano, L. R., Herrera, G., Jordan, R. y Gimeno, J. (2010). PictogramRoom: uso de la Realidad Aumentada para el aprendizaje visual de las personas con Autismo, en M. Belinchón (coord.), Investigaciones sobre el autismo en español: problemas y perspectivas (pp. 437-448). Madrid: Centro de Psicología Aplicada de la Universidad Autónoma de Madrid (CPAUAM).

Fleury, V. P. y Schwartz, I. S. (2017). A Modified Dialogic Reading Intervention for Preschool Children With Autism Spectrum Disorder. Topics in Early Childhood Special Education 37(1), 16-28.

Fleury, V., Herriott-Miramontez, S., Hudson, R. y Schwartz, I. (2014). Promoting active participation in book reading for preschoolers with Autism Spectrum Disorder: a preliminary study. Child Language Teaching and Therapy 30(3), 273-288.

Freeden, R. y Koegel, R. (2006). The pivotal role of initiations in habilitation, en R. L. Koegel y L. K. Koegel (eds.), Pivotal response treatments for autism: Communication, social, \& academic development (pp. 165-188). Baltimore, MD: Brookes Publishing.

Goldstein, H. (2002). Communication intervention for children with autism: a review of treatment efficacy. Journal of Autism and Developmental Disorders 32(5), 373-396.

González, A., Llorente, M., Fragoso, J., Martos, J., Ayuda, R. y Freire, S. (2014). Trastornos del espectro autista de alto funcionamiento. Otra forma de aprender. Madrid: CEPE.

Happé, F. y Frith, U. (2006). The weak coherence account: detail-focused cognitive style in autism spectrum disorders. Journal of Autism and Developmental Disorders 36(1), 5-25.

Hudson, M. y Test, D. (2011). Evaluating the evidence base of shared story reading to promote literacy for students with extensive support needs. Research \& Practice for Persons with Severe Disabilities 36(1-2), 34-35. 
Justice, L. M., Mashburn, A. J., Hamre, B. K. y Pianta, R. C. (2008). Quality of language and literacy instruction in preschool classrooms serving atrisk pupils. Early Childhood Research Quarterly 23(1), 51-68.

Karamanoli, P., Tsinakos, A. y Karagiannidis, C. (2017). The application of augmented reality for intervention to people with Autism, Spectrum Disorders. Journal of Mobile Computing \& Application 4(2), 42-51.

Lanter, E., Watson, L., Erickson, A. y Freeman, D. (2012). Emergent literacy in children with autism: an exploration of developmental and contextual dynamic processes. Language, Speech, and Hearing Services in Schools 43(3), 308-324.

Lin, F. y Chaо J.-T. (2010) Augmented Reality-Based Assistive Technology for Handicapped Children. International Symposium on Computer, Communication, Control and Automation (3CA), Tainan (pp. 61-64).

Mucchetti, C. A. (2013). Adapted shared reading at school for minimally verbal students with autism. Autism 17(3), 358-372.

National Early Literacy Panel (2008). Developing early literacy: Report of the National Early Literacy Panel. Washington, DC: National Institute for Literacy.

Ozonoff, S., Pennington, B. F. y Rogers, S. J. (1991). Executive Function Deficits in High-Functioning Autistic Individuals: Relationship to Theory of Mind. Journal of Child Psychology and Psychiatry 32(7), 1081-1105.

Penimonti, J. M. y Justice, L. M. (2010). Teachers' use of scaffolding strategies during read alouds in the preschool classroom. Early Childhood Education Journal 37, 241-248.

Stein, N. L. y GlenN, C. (1979). An analysis of story comprehension in elementary school children, en R. O. Freedle (ed.), New directions in discourse processing. Vol. 2: Advances in discourse processing (pp. 53-120). Norwood, NJ: Ablex.

Stirling, L., Douglas, S., Leekam, S. y Carey, L. (2014). The use of narrative in studying communication in Autism Spectrum Disorders: A review of methodologies and findings, en J. Arciuli \& J. Brock (eds.), Communication in autism. Trends in language adquisition research (vol. 11, pp. 171215). Ámsterdam: John Benjamins. 
Tager-Flusberg, H., Paul, R. y Lord, C. (2005). Language and Communication in Autism, en F. R. Volkmar, R. Paul, A. Klin y D. Cohen (eds.), Handbook of autism and pervasive developmental disorders: Diagnosis, development, neurobiology, and behavior (pp. 335-364). Hoboken, NJ, US: John Wiley \& Sons Inc.

Teale, W. H. y Sulzby, E. (1986). Emergent Literacy as a Perspective for Examining How Young Children Become Writers and Readers. Norwood, NJ: Ablex.

Thaut, M. H. (1987). Visual versus auditory (musical) stimulus preferences in autistic children: a pilot study. Journal of Autism and Developmental Disorders 17(3), 425-432.

van Kleeck, A., Vander Woude, J. y Hammett, L. (2006). Fostering literal and inferential language skills in Head Start preschoolers with language impairment using scripted book-sharing discussions. American Journal of Speech Language Pathology 15(1), 85-95.

Watson, L. R., Andrews, M. D. y Orovitz, J. (1996). Emergent literacy in children with autism vs. typical development. Comunicación presentada en la reunión de la American Speech-Language-Hearing Association, Seattle, WA.

Wetherby, A., Prizant, B. y Schuler, A. (2000). Understanding the nature of the communication and language impairments, en A. Wetherby y B. Prizant (eds.), Autism spectrum disorders: A transactional developmental perspective (pp. 109-141). Baltimore: Paul H. Brookes.

Wetherby, A. y Prizant, B. (2002). Communication and Symbolic Behavior Scales Developmental Profile (CSBS DP). Baltimore, MD: Brookes Publishing.

Whalon, K, Delano, M. y Hanline, M. F. (2013). A Rationale and Strategy for Adapting Dialogic Reading for Children with Autism Spectrum Disorder: RECALL. Preventing School Failure 57(2), 93-101.

Whalon, K., Martínez, J., Shannon, D., Butcher, C. y Hanline, M. F. (2015). The impact of reading to engage children with autism in language and learning (RECALL). Topics in Early Childhood Education 35(2), 102-115. Whitehurst, G. J., Arnold, D. S., Epstein, J. N., Angell, A. L., Smith, M. y FisCHEL, J. E. (1994). A picture book reading intervention in day care 
and home for children from low-income families. Developmental psychology 30(5), 679.

Zevenbergen, A. A. y Whitehurst, G. J. (2003). Dialogic reading: A shared picture book reading intervention for preschoolers, en A. van Kleeck, S. A. Stahl y E. B. Bauer (eds.), On reading books to children (pp. 177-200). Nueva York, NY: Routledge. 